\title{
The first XMM-Newton spectrum of a high redshift quasar - PKS 0537-286
}

\author{
J. N. Reeves ${ }^{1}$, M. J. L. Turner ${ }^{1}$, P. J. Bennie ${ }^{1}$, K. A. Pounds ${ }^{1}$, A. Short ${ }^{1}$, P. T. O’Brien ${ }^{1}$, Th. Boller ${ }^{2}$ \\ M. Kuster ${ }^{3}$, and A. Tiengo ${ }^{4}$
}

1 X-Ray Astronomy Group, Department of Physics and Astronomy, Leicester University, Leicester LE1 7RH, UK

2 Max-Planck-Institut für extraterrestrische Physik, Postfach 1603, 85748 Garching, Germany

3 Institut für Astronomie und Astrophysik - Astronomie, University of Tübingen, Waldhäuser Strasse 64, 72076 Tübingen, Germany

4 IFC Milan, 20133 Milano, Italy

Received 2 October 2000 / Accepted 17 October 2000

\begin{abstract}
We present XMM-Newton observations of the high redshift $(z=3.104)$, radio-loud quasar PKS 0537-286. The EPIC CCD cameras provide the highest signal-to-noise spectrum of a high- $z$ quasar to date. The EPIC observations show that PKS 0537-286 is extremely X-ray luminous $\left(L_{\mathrm{X}}=210^{47} \mathrm{erg} \mathrm{s}^{-1}\right)$, with an unusually hard X-ray spectrum $(\Gamma=1.27 \pm 0.02)$. The flat power-law emission extends over the whole observed energy range ( 0.4 to $40 \mathrm{keV}$ in the quasar rest frame); there is no evidence of intrinsic absorption, which has been claimed in PKS 0537-286 and other high $z$ quasars. However, there is evidence for weak Compton reflection. A redshifted iron $\mathrm{K}$ line, observed at $1.5 \mathrm{keV}$ - corresponding to $\sim 6.15 \mathrm{keV}$ in the quasar rest frame - is detected at $95 \%$ confidence. If confirmed, this is the most distant iron $\mathrm{K}$ line known. The line equivalent width is small $(33 \mathrm{eV})$, consistent with the "X-ray Baldwin effect" observed in other luminous quasars. The reflected continuum is also weak $(R \lesssim 0.25)$. We find the overall spectral energy distribution of PKS $0537-286$ is dominated by the $\mathrm{X}$-ray emission, which, together with the flat power-law and weak reflection features, suggests that the X-radiation from PKS 0537-286 is dominated by inverse Compton emission associated with a face-on relativistic jet.
\end{abstract}

Key words. galaxies: active - quasars: individual: PKS 0537-286 - X-rays: galaxies

\section{Introduction}

At the present time there is little known about the X-ray spectra of high redshift quasars, and thus a lack of understanding of the nature of the quasar central engine at the highest redshifts. However the situation is improving; the ROSAT mission substantially increased the number of high $z$ quasars with detected X-ray emission (Brinkmann et al. 1997), whilst $A S C A$ obtained the first broad-band spectra (e.g. Cappi et al. 1997; Reeves et al. 1997; Vignali et al. 1999), albeit with rather limited photon statistics and sensitivity. The high throughput and bandpass of $X M M$-Newton is about to revolutionize studies of high redshift quasars. Many high redshift quasars should be found routinely through follow-up surveys of XMM fields (Watson et al. 2001), detailed spectral studies can be performed on the brightest objects and the high sensitivity limit of $X M M$-Newton should enable the detection of the most distant known quasars at $z>5$ (Brandt et al. 2000).

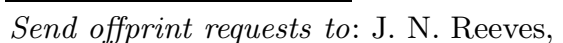
e-mail: jnr@star.le.ac.uk
At $z=3.104$, PKS $0537-286$ is one of the brightest known high $z$ quasars, first recognised as a 1 Jy Parkes southern radio source (e.g. Bolton et al. 1975) and later identified as a QSO (Wright et al. 1978). The optical (rest frame UV) spectrum shows two strong absorption systems associated with the quasar, one of which produces a cut-off in the quasar continuum at the Lyman-limit. The redshift of PKS 0537-286 has been confirmed at $z=3.104 \pm 0.001$ (Osmer et al. 1994). PKS 0537-286 was first detected as an X-ray source by the Einstein observatory (Zamorani et al. 1981), with a position within $1^{\prime}$ of the radio and optical source. The identification was later confirmed by ROSAT (Bühler et al. 1995), and with $A S C A$ there were enough counts to obtain a crude X-ray spectrum (Siebert et al. 1996; Cappi et al. 1997; Reeves et al. 1997). These observations revealed a luminous X-ray source, with a flat X-ray spectrum perhaps indicative of a soft X-ray cut-off due to absorption. We now present the $X M M-N e w t o n$ observations of PKS 0537-286. Values of $H_{0}=50 \mathrm{~km} \mathrm{~s}^{-1} \mathrm{Mpc}^{-1}$ and $q_{0}=0.5$ have been assumed and all fit parameters are given in the quasar rest-frame. 


\section{XMM-Newton observations}

PKS 0537-286 was observed during orbit 51 of the Cal$\mathrm{PV}$ phase of XMM-Newton. Processing was performed using the XMM SAS (Science Analysis Software). The initial data files were processed with the EMCHAIN and EPCHAIN scripts, using the latest known calibration. X-ray events corresponding to patterns $0-12$ for the 2 MOS cameras (similar to grades $0-4$ in $A S C A$ ) were used; for the $\mathrm{PN}$, only pattern 0 events (single pixel events) were selected. For the MOS detectors, electronic noise was screened out by rejecting events with negative E3 values. Known hot or bad pixels were also removed during screening. We refer the reader to Turner et al. (2001) for an in-depth description of the EPIC-MOS instruments, whilst a detailed account of the EPIC-PN detector can be found in Strüder et al. (2001). The non X-ray background remained relatively low throughout the observations, so it was not necessary to filter events on time. We also found no variability in the source count rate during the observation.

The screening process yielded $19.2 \mathrm{ksec}$ of data for MOS-1, $28.0 \mathrm{ksec}$ for MOS-2 and $38 \mathrm{ksec}$ for the PN detector. Source spectra were extracted from circular regions of $30^{\prime \prime}$ radius for the $\mathrm{PN}$ (to avoid the edge of the chip) and $1^{\prime}$ radius for the MOS. Background spectra were taken from an identical circular region, offset from the source position. The majority of source counts fall onto these source regions (at least $80 \%$ for the $\mathrm{PN}$ and $>90 \%$ for the MOS), whilst the background count rates are very low; $1.07 \pm 0.1710^{-2}$ cts s$^{-1}, 4.8 \pm 1.510^{-3}$ cts s$^{-1}$ and $8.8 \pm 1.110^{-3}$ cts s $^{-1}$ for the PN, MOS-1 and MOS-2 respectively. The net source count rates obtained are $0.257 \pm$ $0.004 \mathrm{cts} / \mathrm{s}, 0.268 \pm 0.003 \mathrm{cts} / \mathrm{s}$ and $0.513 \pm 0.004 \mathrm{cts} / \mathrm{s}$, for MOS-1, MOS-2 and the PN respectively. At this level, even in full-frame mode, photon pile-up is negligible. We do not consider the RGS data in this paper, as the count rate is too low $(<0.1 \mathrm{cts} / \mathrm{s})$ for a reliable analysis of the dispersed spectrum. The background subtracted EPIC spectra were fitted, using XSPEC V11.0, with the latest response matrices produced by the EPIC team; the systematic level of uncertainty is $<5 \%$. Spectra were binned to a minimum of 20 counts per bin, to apply the $\chi^{2}$ minimisation technique. All subsequent errors are quoted at $90 \%$ confidence $\left(\Delta \chi^{2}=4.6\right.$ for 2 interesting parameters $)$.

\section{EPIC spectral analysis}

We initially fitted the individual X-ray spectra from the 3 EPIC detectors, to check for consistency between the datasets. From a simple power-law fit it was found that the photon index agreed for all 3 detectors $(\Gamma=1.25-$ 1.30) and that the relative normalisations were consistent to within $5 \%$. Therefore we proceeded to fit all three datasets simultaneously, assuming a Galactic column of $N_{\mathrm{H}}=1.9510^{20} \mathrm{~cm}^{-2}$ (Elvis et al. 1989). The EPIC spectrum is plotted in Fig. 1 and the results from a power-law fit to the broad-band data are shown as fit 1 (in Table 1). Clearly a power-law is a good fit $\left(\chi_{\nu}^{2} \sim 1\right)$, with no obvious

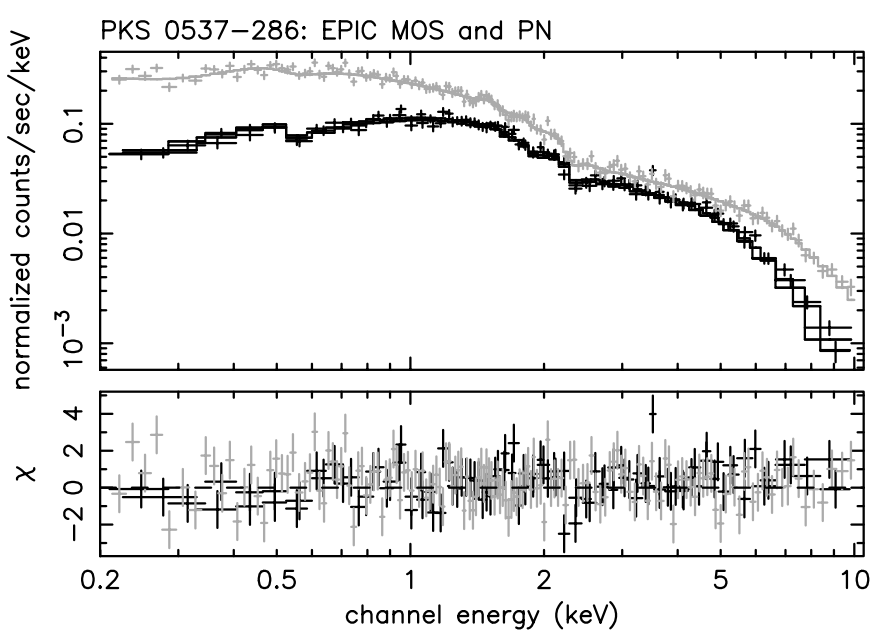

Fig. 1. EPIC-MOS and EPIC-PN (greyscale) spectra of the quasar PKS 0537-286. The spectrum corresponds to fit 2 in Table 1 (power-law with iron line and $\mathrm{K}$ edge). The flat $(\Gamma=$ 1.27) power-law extends over the whole QSO energy range, from $0.8-40 \mathrm{keV}$, with no sign of a low energy cut-off

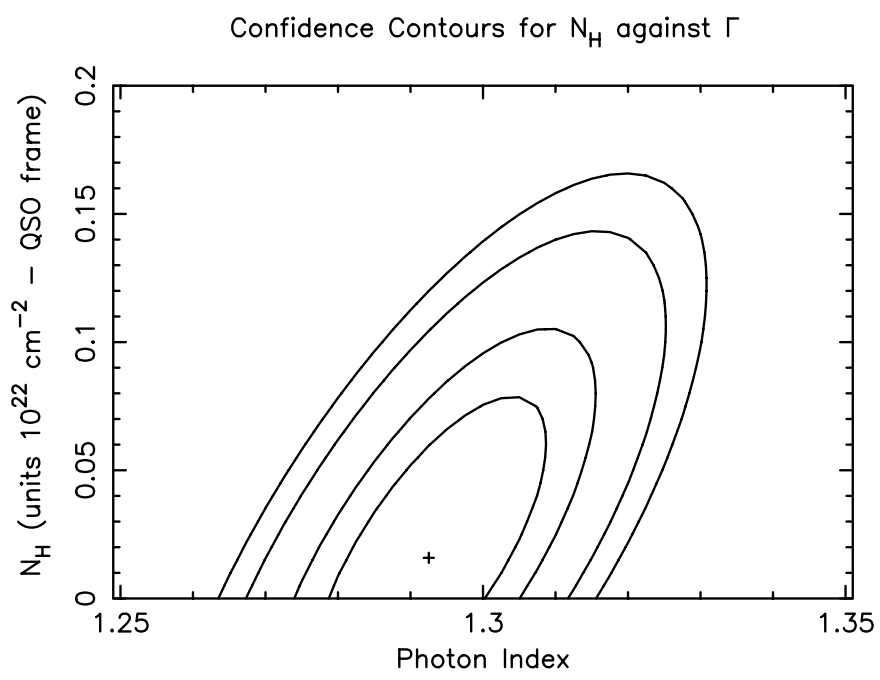

Fig. 2. A confidence contour plot, showing the quasar photon index against the column density. Contours represent the $68 \%$, $90 \%, 99 \%$ and $99.9 \%$ levels. There is no X-ray absorption in PKS 0537-286 down to a limit of $110^{21} \mathrm{~cm}^{-2}$ in the QSO frame (at $90 \%$ confidence)

continuum curvature present. We note that the power-law photon index of $\Gamma=1.27 \pm 0.02$ is considerably flatter than the mean quasar index (e.g. Reeves \& Turner 2000), even for radio-loud quasars which have a typical mean of $\Gamma=1.6$. The observed broad-band $(0.2-10 \mathrm{keV})$ flux is $3.110^{-12} \mathrm{erg} \mathrm{cm}^{-2} \mathrm{~s}^{-1}$, which, under the assumption of isotropic X-ray emission, corresponds to a luminosity at $z=3.104$ of $2.210^{47} \mathrm{erg} \mathrm{s}^{-1}(0.8-40 \mathrm{keV}$ in the QSO frame). This makes PKS 0537-286 one of the most luminous known quasars in the Universe.

We also searched the spectrum for signs of photoelectric absorption. There are several reported cases in the 
PN and MOS Fe K Profile for PKS 0537-286

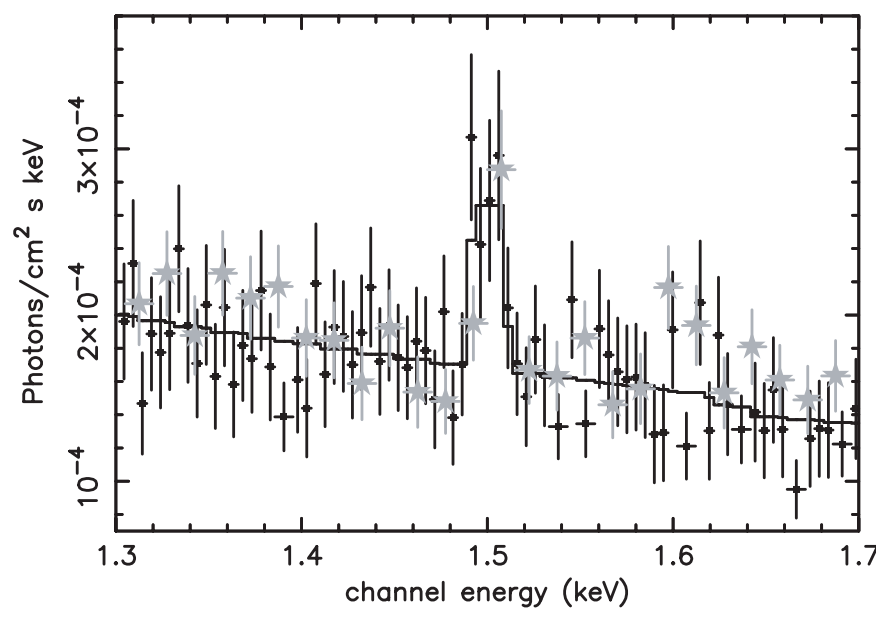

Fig. 3. A close-up of the EPIC PN and MOS (greyscale) data between $1.3-1.7 \mathrm{keV}$. The iron $\mathrm{K}$ emission line, at $z=3.104$, is redshifted down to $1.5 \mathrm{keV}$ in the observed spectrum. The MOS-1 and MOS-2 data have been co-added

literature of excess X-ray absorption towards high $z$ quasars, from ROSAT and ASCA observations (see Sect. 4). The excellent soft X-ray response of the EPIC instruments allows us to place very tight limits on the amount of obscuring material. However we found no excess absorption towards PKS 0537-286, the power-law extending un-broken down to $0.2 \mathrm{keV}$. A confidence contour plot is shown as Fig. 2; we place a $90 \%$ upper limit on the intrinsic column of $N_{\mathrm{H}}<5.010^{19} \mathrm{~cm}^{-2}$, which in the quasar rest frame (at $z=3.104$ ) corresponds to $N_{\mathrm{H}}<1.0$ $10^{21} \mathrm{~cm}^{-2}$.

\subsection{The iron $\mathrm{K}$ emission properties}

The relative brightness of PKS 0537-286 allows us, for the first time, to search for signs of the iron $\mathrm{K} \alpha$ emission line in a high redshift quasar. We restricted our search to the $1-2 \mathrm{keV}$ band, where the iron $\mathrm{K}$ line should appear at this redshift; conveniently the throughput of the $X M M$-Newton EPIC instrument reaches a maximum in this band. We initially fixed the line width at $\sigma=10 \mathrm{eV}$ and the result is shown as fit 2 . A narrow $\mathrm{Fe} \mathrm{K}$ line is detected (equivalent width $E W=33 \mathrm{eV}$, QSO frame), at $95 \%$ confidence for 2 parameters, whilst the line peaks at $6.15 \pm 0.15 \mathrm{keV}$ (or $1.5 \mathrm{keV}$ observed-frame). A close up of the EPIC PN and MOS data, illustrating the line detection at $z=3.104$, is shown in Fig. 3. The confidence contours to the line, from a fit to the MOS and PN data, are also shown in Fig. 4. Although the line energy is consistent with neutral material, the apparent redshift of the line may indicate an origin from the inner quasar accretion disk (Fabian et al. 1989) or from reprocessing in infalling matter. The velocity width of the line is not well constrained, with an upper limit of $\sigma<500 \mathrm{eV}$. The line emission is considerably weaker than the Fe lines

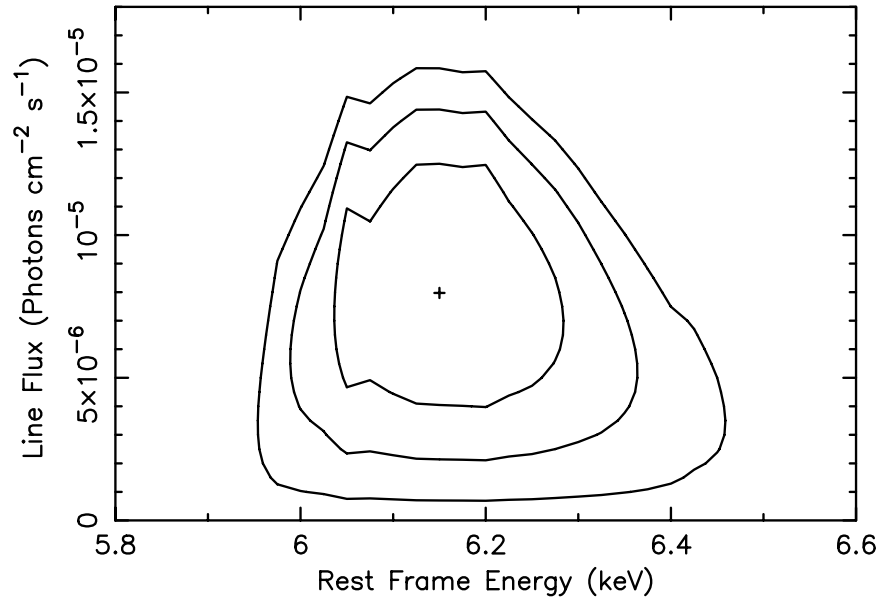

Fig. 4. Confidence contours for the iron $\mathrm{K}$ line in PKS 0537-286. Contours represent the $68 \%, 90 \%$ and $95 \%$ levels. The Fe K line is detected at $95 \%$ confidence. Note the slight redshift of the line energy

observed in Seyfert 1 galaxies (Nandra et al. 1997) and in some radio-quiet quasars (Reeves \& Turner 2000).

Finally we tried to constrain the iron $\mathrm{K}$ absorption edge, which in disk reflection models (e.g. George \& Fabian 1991) is predicted to accompany the iron K line. We fixed the edge energy at $7.1 \mathrm{keV}$, consistent with neutral iron. We obtained a value of $\tau=0.05_{-0.04}^{+0.05}$, although the edge is only detected at $90 \%$ confidence (see fit 2 ).

\subsection{The effect of aluminium $K \alpha$ on the iron line}

Since the proposed iron $\mathrm{K}$ line (observed at $1.5 \mathrm{keV}$ ) lies near the $\mathrm{Al}-\mathrm{K} \alpha$ line, at $1.487 \mathrm{keV}$, in the detector background, we have checked to see whether this can contribute towards the observed Fe line flux at $1.5 \mathrm{keV}$. To do this we extracted a large source-free background region, of $4^{\prime}$ radius, from the PKS $0537-286$ PN field, and then measured the strength of the $\mathrm{Al} \mathrm{K} \alpha$ line directly. We renormalised the background fluxes (from an area 63 times larger), to the size of our source extraction region. It was found that the total integrated count rate from the $\mathrm{Al} \mathrm{K} \alpha$ line was $7.5 \pm 2.610^{-5} \mathrm{ct} \mathrm{s}^{-1}$, compared to the quasar line count rate of $1.85 \pm 0.610^{-3} \mathrm{ct} \mathrm{s}^{-1}$. Thus we can conclude that the $\mathrm{Al} \mathrm{K} \alpha$ line contributes only $\sim 4 \%$ of the quasar line flux, before background subtraction. Note that at $1.5 \mathrm{keV}$, the total background count rate is $<1 \%$ of the total quasar count rate. There are also no strong variations in background, across the central chips, for either the MOS or PN.

As a final check, we also used a long closed-filter PN observation (in orbit 59) to estimate the background count rate from the $\mathrm{Al} \mathrm{K} \alpha$ line. We found that the total flux in the $\mathrm{Al}$ line (for an area the size of the PN source extraction region) was $9.010^{-5} \mathrm{ct} \mathrm{s}^{-1}$, consistent with the value given above, corresponding to only $5 \%$ of the quasar line 
Table 1. X-ray spectral fits to PKS 0537-286. ${ }^{a}$ Rest energy of the iron K line or edge in keV (QSO frame). ${ }^{b}$ Equivalent width of the line in $\mathrm{eV} .{ }^{c}$ Optical depth of the iron $\mathrm{K}$ edge. ${ }^{d}$ Strength of the reflection component $R(=\Omega / 2 \pi)$. ${ }^{e}$ Assumed value for high energy cut-off in $\mathrm{keV} .{ }^{f}$ Indicates parameter is fixed

\begin{tabular}{|c|c|c|c|c|c|c|c|c|c|}
\hline \multirow[t]{2}{*}{ Fit } & \multirow[t]{2}{*}{ Model } & \multirow[t]{2}{*}{$\Gamma$} & \multicolumn{2}{|c|}{ Iron line } & \multicolumn{2}{|c|}{ Iron edge } & \multicolumn{2}{|c|}{ Reflection } & \multirow[t]{2}{*}{$\chi^{2} /$ dof } \\
\hline & & & $E^{a}$ & $E W^{b}$ & $E^{a}$ & $\tau^{c}$ & $R^{d}$ & $E_{\mathrm{c}}^{e}$ & \\
\hline 1. & PL only & $1.27 \pm 0.02$ & & & & & & & $1049.2 / 1000$ \\
\hline 2. & $\mathrm{PL}+\mathrm{GA}+\mathrm{EDGE}$ & $1.27 \pm 0.02$ & $6.15 \pm 0.15$ & $33_{-23}^{+30}$ & $7.1^{f}$ & $0.05_{-0.04}^{+0.05}$ & & & $1038.5 / 997$ \\
\hline 3. & $\mathrm{PL}+\mathrm{GA}+\mathrm{PEXRAV}$ & $1.28 \pm 0.04$ & $6.15^{f}$ & $33^{f}$ & & & $0.25_{-0.09}^{+0.11}$ & $100^{f}$ & $1044.5 / 998$ \\
\hline 4. & $\mathrm{PL}+\mathrm{GA}+$ PEXRAV & $1.29 \pm 0.04$ & $6.15^{f}$ & $33^{f}$ & & & $0.11_{-0.08}^{+0.09}$ & $250^{f}$ & $1038.6 / 998$ \\
\hline 5. & $\mathrm{PL}+\mathrm{GA}+$ PEXRAV & $1.30 \pm 0.04$ & $6.15^{f}$ & $33^{f}$ & & & $0.03_{-0.03}^{+0.07}$ & none & 1036.9/998 \\
\hline
\end{tabular}

counts. Therefore it is highly unlikely that the background subtraction at $1.5 \mathrm{keV}$ contributes towards the detection of the quasar iron $\mathrm{K}$ line.

\subsection{Constraints on the Compton reflection continuum}

Having detected these weak iron $\mathrm{K}$ features, we next tried to constrain the properties of the Compton reflection "hump", particularly as the observed emission extends up to $40 \mathrm{keV}$ in the quasar rest frame. We used the PEXRAV model in XSPEC (Magdziarz \& Zdziarski 1995), assuming a disk inclination of $30^{\circ}$ and a high energy cutoff equal to $100 \mathrm{keV}$. We left $R$, the fraction of material (of $2 \pi$ steradian solid angle) that is irradiated by the X-ray source (where $R=\Omega / 2 \pi$ ), as a free parameter in the fits. The results are given as fit 3 . We find a best-fit value for the reflection continuum of $R=0.25_{-0.09}^{+0.11}$, indicating that the reflecting material subtends a solid-angle lower than the $2 \pi$ steradians expected from an accretion disk geometry. The small amount of reflection (with $R \sim 0.25$ ), is consistent with the low equivalent width of the iron $\mathrm{K}$ line.

We note however, that our assumption for the high energy cut-off of the hard X-ray power-law may effect the fits. To test this we tried two additional fits, one with a cut-off at $250 \mathrm{keV}$ and one fit with no high energy cutoff (fits $4-5$ ). For the former we find that the reflection component is even weaker $\left(R=0.11_{-0.08}^{+0.09}\right)$, whilst with no cut-off the value of $R$ is consistent with zero $(R<0.10)$. Comparing the statistics of fits $3-5$, there is a preference for very weak reflection and no high energy cut-off, although the differences are not significant. Finally we tried fixing $R$ at 1 in our fits. In this case, whatever cut-off energy was assumed, the fit obtained was always considerably worse (by $\Delta \chi^{2}>100$, or at $>99.99 \%$ confidence).

\subsection{The spectral energy distribution of PKS 0537-286}

In Fig. 5, we compile all the published multi-wavelength data on PKS 0537-286, together with the XMM-Newton spectrum. The SED (spectral energy distribution) of PKS 0537-286 is extreme when compared to a typical radio-loud quasar (Elvis et al. 1994). In PKS 0537-286 the majority of the observed quasar power is radiated
Radio to X-ray SED of PKS 0537-286

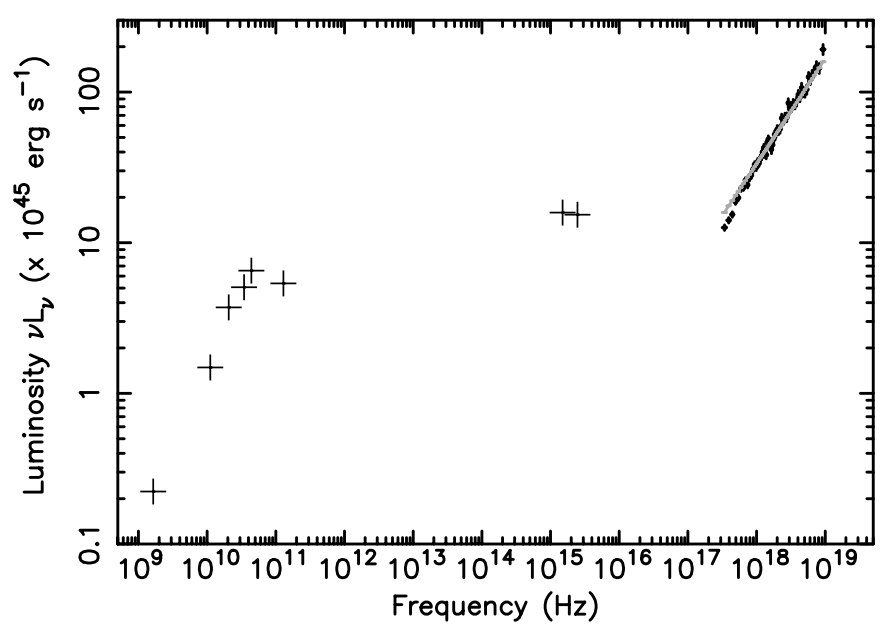

Fig. 5. The radio to X-ray spectral energy distribution of PKS 0537-286. The total power output of the quasar is dominated by the X-ray emission. The SED is similar to other high luminosity blazars at high redshift (see Fabian et al. 1999)

in the X-ray band (typically in AGN the X-ray emission is only $5 \%$ of the bolometric luminosity; e.g. Elvis et al. 1994). The optical-UV emission is also relatively weak in comparison to the X-rays $\left(\alpha_{\mathrm{ox}}=-0.94\right)$. However this is consistent with the observations of other high redshift blazar-like AGN (see Fabian et al. 1999), where a strong, but flat, X-ray emission component is also seen.

\section{Discussion}

The XMM-Newton observations have showed that PKS 0537-286 is an extremely luminous quasar, with a particularly flat X-ray spectrum and weak iron K emission and reflection features. Indeed the total power output of PKS 0537-286 is dominated by the X-ray emission. A likely interpretation is that the X-rays mainly arise through the inverse Compton emission associated with a relativistic jet. In this scenario relativistic electrons (or positrons) in the jet Compton upscatter either the low energy synchrotron photons produced in the jet (Synchrotron Self Compton - Jones et al. 1974; Ghisellini et al. 1985) or alternatively the thermal optical/UV 
photons from the accretion disk (External Inverse Compton - Ghisellini \& Madau 1996). This can naturally account for the hard spectrum of PKS 0537-286, as in the X-ray band we are observing the onset of this Inverse Compton component, presumably extending out to $\gamma$-ray energies.

As it appears that we can rule out a scenario whereby all of the primary X-ray emission originates above the accretion disk, we instead tried to fit the data with two continuum emission components; a "Seyfert-like" steep power-law (with $\Gamma$ fixed at 1.9) originating from near the accretion disk, and a hard, bright jet component with a flat X-ray slope (i.e. $\Gamma=1.2-1.3$ as before). We modified the Seyfert-like power-law with a continuum reflection component (assuming $R=1$ ) and also added a narrow iron $\mathrm{K}$ line of equivalent width $150 \mathrm{eV}$. Although poorly constrained, we obtained a good fit to the data when the Seyfert-like component was typically $4-5$ times weaker than the flat jet component. Thus in PKS 0537-286 we may be observing two X-ray components; the steep Seyfert-like emission from near the disk, responsible for the iron $\mathrm{K}$ line, and the hard component from the jet, which considerably dilutes the disk reflection and line features.

This provides a possible explanation for the apparent weakness of the iron $\mathrm{K}$ line and Compton reflection hump, when compared to other observations of Seyfert galaxies and quasars. If the relativistic jet is orientated close to the line-of-sight, as expected in unification schemes for quasars (Antonucci 1993), then any disk reflection features can be diluted due to Doppler boosting of the featureless X-ray continuum, through the bulk motion of the jet. This is consistent with other observations of X-ray luminous, predominantly radio-loud quasars, where the strength of the iron line is diminished (the so-called "X-ray Baldwin effect" - Iwasawa \& Taniguchi 1993). For PKS 0537-286, at a redshift of $z=3.104$, any iron $K$ emission features are redshifted into a more favourable part of the EPIC bandpass, where the effective area is near its maximum. This has enabled us to detect a weak iron $K$ line in PKS 0537-286; indeed at $z=3.104$ this is the most distant iron $K$ emission line detected to date. The limited statistics do not allow the line width or shape to be constrained. However, the median energy of $6.15 \mathrm{keV}$ implies reprocessing in "cold" matter close to the central black hole or from infalling material at $v \sim 13000 \mathrm{~km} \mathrm{~s}^{-1}$. Clearly, more detailed studies with XMM-Newton of other high redshift quasars, both radio-loud and radio-quiet, are required to explore this.

Past studies with ROSAT and ASCA have showed that several high redshift radio-loud quasars appear to show intrinsic X-ray absorption, above that from our own Galaxy (see Bechtold et al. 1994; Elvis et al. 1994b; Reeves et al. 1997; Cappi et al. 1997; Fiore et al. 1998). Correlations involving samples of quasars (Reeves et al. 1997; Reeves \& Turner 2000) have showed that this apparent excess absorption is correlated with object redshift, with many high $z$ quasars requiring substantial absorption columns (up to $10^{22} \mathrm{~cm}^{-2}$ ), whilst low $z$ quasars remain unabsorbed. Recently even, evidence has been presented for substantial soft X-ray absorption towards one blazarlike object (GB 1428+4217) at $z=4.71$ (Boller et al. 2000). At present the nature of this absorption is unclear, but it could be related to the dense Galactic-scale environments proposed in the early evolutionary stages of quasars (see Fabian 1999). Alternatively in some objects the X-ray absorption may originate from intervening damped Lyman- $\alpha$ systems, which are thought to be associated with the disks of young protogalaxies (e.g. Wolfe et al. 1994).

From the published optical (rest frame UV) spectrum, it is known that PKS 0537-286 has substantial UV absorption, with at least two different Lyman-limit systems observed close to the known redshift of the quasar (Wright et al. 1978; Osmer et al. 1994). The velocities of these systems are $\Delta \nu=4200$ and $40000 \mathrm{~km} \mathrm{~s}^{-1}$, blueshifted with respect to the quasar. The latter system has a very deep Lyman-continuum cut-off at $912 \AA$ (rest frame), where the quasar continuum flux drops close to zero. So it perhaps seems surprising at first that in this $X M M$ Newton observation we detect no intrinsic X-ray absorption towards PKS 0537-286. However in order to measure appreciable line-of-sight X-ray absorption (of the order $N_{\mathrm{H}} \sim 10^{22} \mathrm{~cm}^{-2}$ ), one would have to encounter a damped Lyman- $\alpha$ system. We can now rule this scenario out in PKS 0537-286, and note the chance coincidence of such a system out to $z=3$ is $\sim 20-30 \%$ (e.g. Steidel 1992).

The XMM-Newton observation is in contrast to some earlier ASCA and ROSAT observations of PKS 0537-286, where a low energy X-ray cut-off had been claimed (Reeves et al. 1997; Cappi et al. 1997). One possibility is that the absorption is variable; we note the "warm" absorbers observed in low $z$ AGN are known to vary (e.g. MCG-6-3015, Otani et al. 1996; NGC 3227, George et al. 1998). It is also possible that if the absorption is associated with a high velocity system, the absorbing material may move out of the line-of-sight. Finally, the photon statistics on the earlier ASCA and ROSAT observations were quite poor, so it is possible that the earlier detection was spurious. We conclude that it will be particularly important to utilise future XMM-Newton observations to estimate the frequency of these "absorbed quasars", as a probe of some of the most distant matter in the Universe, and to determine the environments of some of the earliest quasars.

Acknowledgements. This paper is based on observations obtained with XMM-Newton, an ESA science mission with instruments and contributions directly funded by ESA Member States and the USA (NASA). EPIC was developed by the EPIC Consortium led by the Principal Investigator, Dr. M. J. L. Turner. The consortium comprises the following Institutes: University of Leicester, University of Birmingham, (UK); CEA/Saclay, IAS Orsay, CESR Toulouse, (France); IAAP Tuebingen, MPE Garching, (Germany); IFC Milan, ITESRE Bologna, OAPA Palermo, Italy. EPIC is funded by: PPARC, CEA, CNES, DLR and ASI.

We would like to thank the EPIC instrument team, for their hard work during the calibration phase and the SOC 
and SSC teams for making the observation and subsequent analysis possible. We also thank the anonymous referee for his/her prompt response and helpful suggestions.

\section{References}

Antonucci, R. 1993, ARA\&A, 31, 473

Bechtold, J., et al. 1994, AJ, 108, 759

Boller, T., Fabian, A. C., Brandt, W. N., \& Freyberg, M. J. 2000, MNRAS, 315, L23

Bolton, J. G., Shimmins, A. J., \& Wall, J. V. 1975, Aust. J. Phys. Ap. Suppl., 34, 1

Brandt, W. N., Guainazzi, M., Kaspi, S., et al. 2000, AJ, in press [astro-ph/0010328]

Brinkmann, W., Yuan, W., Siebert, J. 1997, A\&A, 319, 413

Bühler, P., Courvoisier, T. J. L., Staubert, R., Brunner, H., \& Lamer, G. 1995, A\&A, 295, 309

Cappi, M., Matsuoka, M., Comastri, A., et al. 1997, ApJ, 478, 492

Elvis, M., Lockman, F. J., \& Wilkes, B. J. 1989, ApJ, 97, 777

Elvis, M., et al. 1994, ApJS, 95, 1

Elvis, M., Fiore, F., Wilkes, B. J., McDowell, J., \& Bechtold, J. 1994b, ApJ, 422, 60

Fabian, A. C., Rees, M. J., Stella, L., \& White, N. E. 1989, MNRAS, 238, 729

Fabian, A. C., et al. 1999, MNRAS, 308, L6

Fabian, A. C. 1999, MNRAS, 308, L39

Fiore, F., Elvis, M., Giommi, P., \& Padovani, P. 1998, ApJ, 492,79
George, I. M., \& Fabian, A. C. 1991, MNRAS, 249, 352

George, I. M., Mushotzky, R., Turner, T. J., et al. 1998, ApJ, 509,146

Ghisellini, G., Maraschi, L., \& Treves, A. 1985, A\&A, 146, 204

Ghisellini, G., \& Madau, P. 1996, MNRAS, 280, 67

Iwasawa, K., \& Taniguchi, Y. 1993, ApJ, 413, L15

Jones, T. W., O'Dell, S. L., \& Stein, W. A. 1974, ApJ, 188, 353

Magdziarz, P., \& Zdziarski, A. A. 1995, MNRAS, 273, 837

Nandra, K., George, I. M., Mushotzky, R. F., Turner, T. J., \& Yaqoob, T. 1997, ApJ, 477, 602

Osmer, P. S., Porter, A. C., \& Green, R. F. 1994, ApJ, 436, 678

Otani, C., et al. 1996, PASJ, 48, 211

Reeves, J. N., Turner, M. J. L., Ohashi, T., \& Kii, T. 1997, MNRAS, 292, 468

Reeves, J. N., \& Turner, M. J. L. 2000, MNRAS, 316, 234

Siebert, J., Matsuoka, M., Brinkmann, W., et al. 1996, A\&A, 307,8

Steidel, C. C. 1992, PASP, 104, 843

Strüder, L., Briel, U., Dennerl, K., et al. 2001, A\&A, 365, L18

Turner, M. J. L., Abbey, A., Arnaud, M., et al. 2001, A\&A, 365, L27

Vignali, C., Comastri, A., Cappi, M., et al. 1999, ApJ, 516, 582

Watson, M. G., Auguères J.-L., Ballet, J., et al. 2001, A\&A, 365, L51

Wolfe, A. M., Fan, X. M, Tytler, D, et al. 1994, ApJ, 435, L101

Wright, A. E., Peterson, B. A., Jauncey, D. L., \& Condon J. J. 1978, ApJ, 226, L61

Zamorani, G., et al. 1981, ApJ, 245, 357 